\title{
The Additional Value of SPECT/CT in Lymphatic Mapping in Breast Cancer and Melanoma
}

\author{
Iris M.C. van der Ploeg ${ }^{1}$, Renato A. Valdés Olmos², Omgo E. Nieweg ${ }^{1}$, Emiel J.Th. Rutgers ${ }^{1}$, Bin B.R. Kroon ${ }^{1}$, and \\ Cornelis A. Hoefnagel ${ }^{2}$ \\ ${ }^{I}$ Department of Surgery, The Netherlands Cancer Institute-Antoni van Leeuwenhoek Hospital, Amsterdam, The Netherlands; and \\ ${ }^{2}$ Department of Nuclear Medicine, The Netherlands Cancer Institute-Antoni van Leeuwenhoek Hospital, Amsterdam, The Netherlands
}

\begin{abstract}
The recently introduced SPECT/CT integrates the physiologic data of SPECT with the anatomic data of CT into a single image. The purpose of this pilot study was to explore the additional value of SPECT/CT in breast cancer patients and melanoma patients with inconclusive planar image findings. Methods: Thirty-one patients had planar lymphoscintigrams showing unexpected lymphatic drainage, 6 had lymphoscintigrams that were difficult to interpret, and 3 showed no drainage on planar imaging. SPECT/CT was performed immediately after delayed planar imaging. Results: In 4 patients, SPECT/CT showed 6 additional sentinel nodes, of which 2 were tumor-positive and led to upstaging and tailored management in $5 \%$ of patients. SPECT/ CT depicted sentinel nodes in 3 patients whose delayed planar imaging had shown no drainage. Conclusion: SPECT/CT was of additional value in finding the exact anatomic location of sentinel nodes in patients with inconclusive planar image findings. SPECT/CT also detected sentinel nodes in addition to those found on planar images, and SPECT/CT detected sentinel nodes in patients whose planar images had shown none.
\end{abstract}

Key Words: radionuclide imaging; breast neoplasms; melanoma; sentinel lymph node biopsy; single-photon emission computed tomography

\section{J Nucl Med 2007; 48:1756-1760}

DOI: 10.2967/jnumed.107.043372

\section{$\mathbf{P}$} lanar lymphoscintigraphy visualizes the sentinel nodes in most patients with breast cancer or melanoma. In some patients, the precise location of a sentinel node may be difficult to determine because of an unexpected lymphatic drainage pattern $(l)$.

SPECT can overcome some of these limitations because of its better contrast and resolution, but SPECT images lack the anatomic detail necessary to create a meaningful surgical roadmap. Several investigators have fused SPECT images with separately acquired CT images to address this

\footnotetext{
Received May 7, 2007; revision accepted Jul. 31, 2007.

For correspondence or reprints contact: Iris M.C. van der Ploeg, MD, Department of Surgery, The Netherlands Cancer Institute-Antoni van Leeuwenhoek Hospital, Plesmanlaan 121, 1066 CX Amsterdam, The Netherlands.

E-mail: i.vd.ploeg@nki.nl

COPYRIGHT (c) 2007 by the Society of Nuclear Medicine, Inc.
}

issue $(1,2)$. The fusion of independently performed SPECT and CT has proved to be complicated because of the need for special computer software with external and internal landmarks. Differences in patient positioning and movement of internal organs between the 2 studies may also cause errors in their alignment.

Recently, a hybrid imaging method consisting of both a SPECT camera and a CT scanner in a single device was introduced. The patient's position does not change between studies, and fusion of the 2 images into 1 image is easier $(1,2)$. Furthermore, corrections for attenuation and scatter have led to improved sentinel node visualization on SPECT. Several authors have reported successful introduction of the SPECT/CT technique in lymphatic mapping in several clinical situations (3-12).

The purpose of this study was to explore the additional value of SPECT/CT in breast cancer patients and melanoma patients with inconclusive planar image findings.

\section{MATERIALS AND METHODS}

At The Netherlands Cancer Institute, lymphoscintigraphy is an integral element of lymphatic mapping. Forty patients underwent SPECT/CT after delayed planar imaging. The group consisted of 31 women with breast cancer and 7 men and 2 women with melanoma. Five melanomas were on the trunk and 4 in the head and neck region. The mean age of the patients was $55 \mathrm{y}$. The indications for performing additional SPECT/CT were planar lymphoscintigrams that showed an unusual lymphatic drainage pattern, were difficult to interpret, or showed no drainage (Table 1). The first indication was present in 2 melanoma patients and 29 breast cancer patients. The second was present in 6 melanoma patients. The last was present in 1 melanoma patient and 2 breast cancer patients.

An 80-MBq dose of ${ }^{99 m}$ Tc-nanocolloid (Nanocoll; GE Healthcare) for melanoma patients, or a $120-\mathrm{MBq}$ dose for breast cancer patients, was injected immediately before lymphoscintigraphy. In patients with breast cancer, the tracer was injected into the tumor, and planar images were obtained $10 \mathrm{~min}, 2 \mathrm{~h}$, and $4 \mathrm{~h}$ after radiotracer injection. In patients with melanoma, the radiopharmaceutical was injected intracutaneously in 4 equal depots around the primary tumor or biopsy site; static images were obtained at 15 min and $2 \mathrm{~h}$ and were preceded by a dynamic study of $10 \mathrm{~min}$. A dual-head $\gamma$-camera equipped with low-energy high-resolution collimators (Vertex; Philips) was used. Both anterior and lateral images were routinely acquired, with additional images obtained 


\begin{tabular}{ll}
\hline \multicolumn{1}{c}{ Indication } & \multicolumn{1}{c}{ Including cases of... } \\
\hline Unusual lymphatic drainage patterns & Extraaxillary drainage in breast cancer patients \\
& Drainage to more than one lymph node basin \\
& Drainage not to nearest lymph node basin \\
Lymphatic patterns difficult to interpret & Sentinel lymph node close to injection site \\
& Deeply located sentinel lymph nodes \\
Nonvisualization & No lymphatic drainage on late planar images \\
\hline
\end{tabular}

if needed. In breast cancer patients, the lateral images were acquired with the patient prone, using the hanging-breast technique. A ${ }^{57} \mathrm{Co}$ flood source was placed behind the patient to outline the body contour. The location of any sentinel nodes was marked on the skin with indelible ink.

SPECT/CT images were acquired immediately after the delayed planar images. The SPECT/CT system (Symbia T; Siemens) consisted of a dual-head variable-angle $\gamma$-camera equipped with low-energy high-resolution collimators and a multislice spiral CT component optimized for rapid rotation. The SPECT acquisition $(128 \times 128$ matrix, 60 frames, $25 \mathrm{~s} /$ frame $)$ was performed using $6^{\circ}$ angular steps in a 20-s time frame. For CT $(130 \mathrm{kV}, 17 \mathrm{mAs}, \mathrm{B} 60 \mathrm{~s}$ kernel), 5-mm slices were obtained. After reconstruction, SPECT images were corrected for attenuation and scatter. Both SPECT and CT axial 5-mm slices were generated using an Esoft 2000 application package (Siemens). These were transferred to a PACS after generation of DICOM files. Images were fused using an Osirix Dicom viewer (version 2.7) in a Unix-based operating system (MAC OS X, Power G5; Apple Inc.). Two nuclear medicine physicians evaluated the images. The SPECT/CT images were also analyzed using 2-dimensional orthogonal reslicing in axial, sagittal, and coronal orientations. A maximum-intensityprojection 3-dimensional display was generated to localize sentinel nodes in relation to anatomic structures. The number and location of the sentinel nodes were determined and described after the planar lymphoscintigraphic image acquisition and after the SPECT/CT image acquisition.

The next day, $1 \mathrm{~mL}$ of patent blue dye (Laboratoire Guerbet) was injected at the tumor site immediately before the operation. The dye and a $\gamma$-ray detection probe (Neoprobe; Johnson \& Johnson Medical) were used to identify the sentinel nodes. A sentinel node was defined as a lymph node through which the primary tumor drains directly. All harvested sentinel nodes were examined by the pathologist using step sectioning and both hematoxylin-eosin and immunohistochemistry staining.

\section{RESULTS}

The exact anatomic location of the sentinel node that prompted the use of SPECT/CT was better visualized with this technique than with planar lymphoscintigraphic imaging in all patients (Table 2; Figs. 1-4). The SPECT/CT findings were found valuable by the operating surgeons and specified the surgical approach in almost all cases. In most breast cancer patients, SPECT/CT specified the location of the internal mammary chain nodes-for example, the exact intercostal space or a position underneath a rib (Fig. 1). An example of the additional value of SPECT/CT in a melanoma patient is shown in Figure 2. Planar images suggested a sentinel node on either side of the neck and suggested these to be dorsal. SPECT/CT revealed that each node was, in fact, in the supraclavicular region, thus changing the surgical approach.

Planar imaging visualized 96 sentinel nodes in 37 of the 40 patients (mean, 2.6; range, 1-5). SPECT/CT showed these same nodes plus 6 additional nodes (mean, 2.6; range, $0-6)$ in 4 patients (10\%), 2 with melanoma and 2 with breast cancer. Three (8\%) of these patients did not show lymphatic drainage on planar lymphoscintigrams, even after massaging of the injection site and with use of delayed imaging and an additional dose of the radiopharmaceutical. Five of the 6 extravisualized sentinel nodes were found intraoperatively. Two of the 5 harvested sentinel nodes that were additionally visualized on SPECT/CT were tumorpositive. These 2 involved sentinel nodes visualized only on SPECT/CT, were the only tumor-positive nodes excised in 2 patients (5\%), and led to a change in disease stage and a change in management.

In 7 patients (18\%), 15 additional sentinel nodes that had not been visualized with either planar or SPECT/CT were intraoperatively identified by palpation, by blue dye, or by the $\gamma$-probe and excised. These were mostly sentinel nodes clustered together that seemed 1 node on lymphoscintigraphy. Two of these additionally excised sentinel nodes were tumor-positive, one in a melanoma patient and the other in a patient with breast cancer. In the end, 12 patients (30\%) had at least 1 tumor-positive sentinel node, of which 2 were depicted only on SPECT/CT and 2 were depicted on neither planar imaging nor SPECT/CT.

\section{DISCUSSION}

Planar lymphoscintigraphy is necessary in tracing the direct lymphatic pathway from a primary tumor site to the sentinel lymph node. The combination of dynamic and static planar images obtained at several intervals after radiotracer injection makes possible this tracing of the pathway. Planar lymphoscintigraphic imaging identifies sentinel nodes in more than $98 \%$ of melanoma patients and in more than $95 \%$ of breast cancer patients $(13,14)$. The concept of a single device that can perform both functional and anatomic imaging 
TABLE 2

Sentinel Lymph Node Characteristics

\begin{tabular}{|c|c|c|c|}
\hline $\begin{array}{l}\text { Location of SNs } \\
\text { on SPECT/CT }\end{array}$ & $\begin{array}{l}\text { No. of SNs } \\
\text { per location }\end{array}$ & $\begin{array}{l}\text { No. of SNs only } \\
\text { on SPECT/CT }\end{array}$ & Additional value of SPECT/CT \\
\hline \multicolumn{4}{|l|}{ Breast cancer patients } \\
\hline Axilla & 37 (49\%) & $2^{*}$ & More precise localization, but not always decisive for surgical approach \\
\hline Internal mammary chain & $26(35 \%)$ & 1 & Precise intercostal localization of all SNs, decisive for surgical approach \\
\hline Supraclavicular region & $3(4 \%)$ & - & Precise localization of all SNs, decisive for surgical approach \\
\hline Infraclavicular region & $6(8 \%)$ & - & Precise localization of all SNs, decisive for surgical approach \\
\hline Interpectoral region & $3(4 \%)$ & - & Precise localization of all SNs, decisive for surgical approach \\
\hline Total & $75(100 \%)$ & 3 & - \\
\hline \multicolumn{4}{|l|}{ Melanoma patients } \\
\hline Axilla & $9(33 \%)$ & 2 & More precise localization, but not always decisive for surgical approach \\
\hline Supraclavicular region & $6(22 \%)$ & - & Precise localization of all SNs, decisive for surgical approach \\
\hline Scapular region & $4(15 \%)$ & $1^{*}$ & Precise localization of all SNs, decisive for surgical approach \\
\hline Neck region & $4(15 \%)$ & - & Precise localization of all SNs, decisive for surgical approach \\
\hline Occipital region & $1(4 \%)$ & - & Precise localization of all SNs, decisive for surgical approach \\
\hline Auricular region & $3(11 \%)$ & - & Precise localization of all SNs, decisive for surgical approach \\
\hline Total & $27(100 \%)$ & 3 & - \\
\hline
\end{tabular}

eventually led to the development of hybrid SPECT/CT systems $(15,16)$. Keidar et al. described the technical aspects of SPECT/CT in 2003 (2). Although planar lymphoscintigraphy shows the lymphatic pathway and the sentinel node in most cases, subsequent SPECT/CT better specifies the anatomic location of the node.

The current study showed the additional value of SPECT/CT in determining the exact anatomic location of sentinel nodes in patients with breast cancer or melanoma.

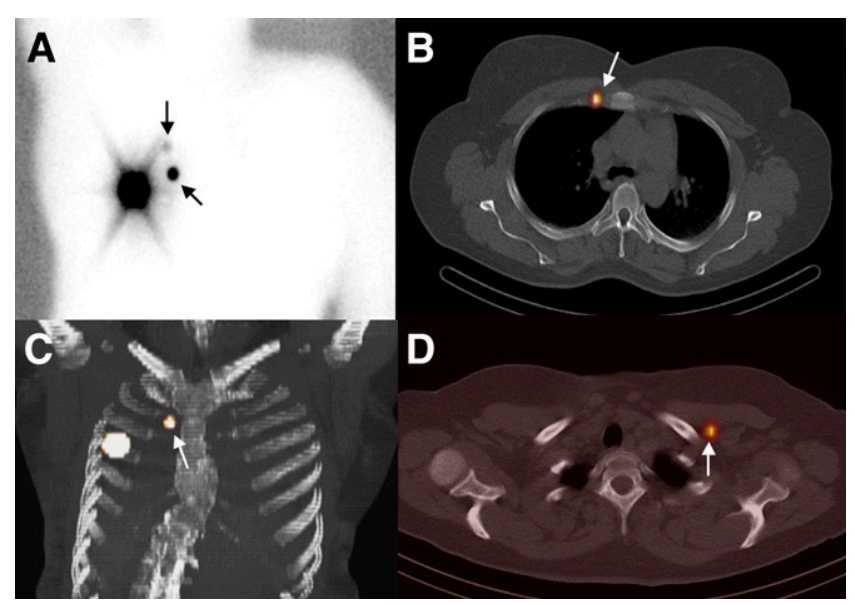

FIGURE 1. (A-C) In woman with right breast cancer, planar lymphoscintigraphy (A) depicts 1 internal mammary chain sentinel node (ascending arrow) with second-echelon node (descending arrow). Axial fused SPECT/CT (B) and 3-dimensional SPECT/CT maximum-intensity projection of thorax $(C)$ enable tracing of sentinel node (arrow), displayed using color wash, underneath rib at second intercostal space close to right border of sternum. (D) In another woman with left breast cancer, axial SPECT/CT fused image visualizes interpectoral sentinel node (arrow).
SPECT/CT remarkably specified the location of the internal mammary chain and other nonaxillary sentinel nodes in breast cancer patients. These nodes are typically small and often accumulate little radioactivity. It may be impossible to locate such a node with a $\gamma$-ray detection probe through the intact skin, making the location of the incision somewhat of

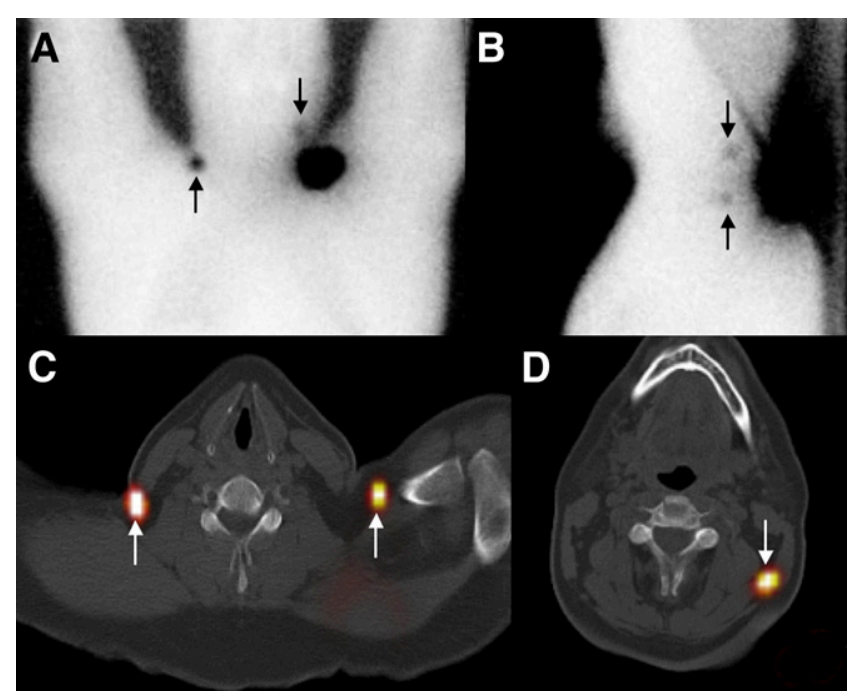

FIGURE 2. Man with melanoma medial to left scapula. (A and B) Planar anterior image and left lateral lymphoscintigram visualize sentinel nodes on either side of neck (ascending arrows) and suggest these to be dorsal. Third sentinel node is depicted cranial to primary tumor site (descending arrows). (C) SPECT/CT shows first 2 nodes (ascending arrows) to be, in fact, located in supraclavicular region on each side. This finding prompted surgeon to place patient supine rather than prone on operating table. (D) SPECT/CT view showing exact anatomic location of third sentinel node (arrow). 


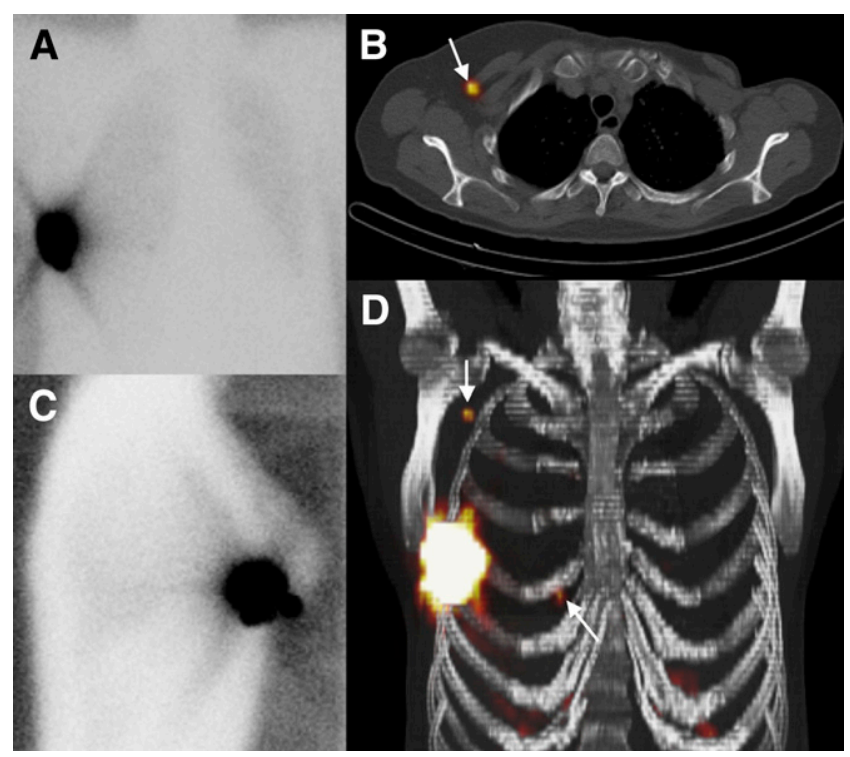

FIGURE 3. Woman with right breast cancer. (A and C) Planar lymphoscintigraphy shows no evident (axillary) drainage on either anterior or lateral image. (B) Axial SPECT/CT fused image shows 1 axillary sentinel node (arrow). (D) On 3-dimensional fused SPECT/CT maximum-intensity projection of thorax, 1 internal mammary sentinel node (ascending arrow) is displayed just below fourth rib.

a gamble. We believe that these nodes are important because their tumor status prompts us to modify subsequent management in $29 \%$ of patients with an internal mammarychain sentinel node and in $18 \%$ of patients with sentinel nodes in other unusual locations $(17,18)$. In melanoma

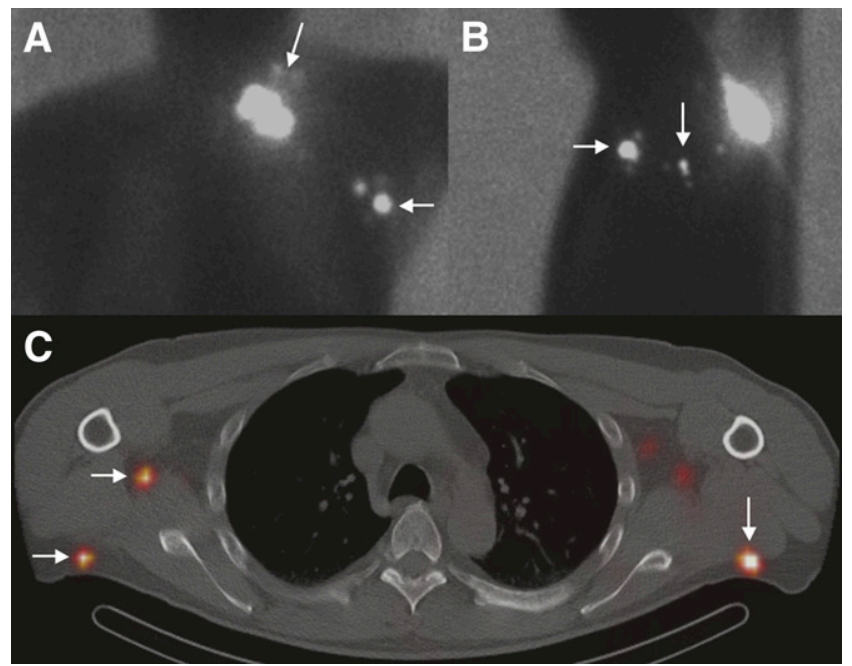

FIGURE 4. Man with melanoma at left scapular region. (A) Anterior planar image depicts sentinel nodes at left supraclavicular (descending arrow) and axillary (horizontal arrow) regions. (B) Lateral planar image also shows sentinel node (descending arrow) in left scapular area. (C) On axial SPECT/CT fused image, not only is left scapular sentinel node (descending arrow) visualized but also another sentinel node (lower horizontal arrow) and a second echelon node (upper horizontal arrow) at right scapular region. Right scapular sentinel node was not visualized on planar images. patients, SPECT/CT is especially helpful in localizing sentinel nodes draining from primary tumors high on the trunk and in the head and neck area. In 4 patients (10\%) in our study, 6 additional sentinel nodes were detected on SPECT/CT, of which 2 were tumor-positive.

The results of our pilot study are similar to what other investigators have found. A review of the literature revealed the use of SPECT/CT in 732 patients with melanoma, breast cancer, head and neck malignancies, and urologic cancers $(3-12,19)$. The traced articles mention rates of sentinel nodes found only on SPECT/CT, and not detected on planar imaging, ranging from $10 \%$ to $30 \%(3,6-9)$. In one of these studies, however, sentinel nodes were detected on planar lymphoscintigraphic imaging but missed on SPECT/CT in $1 \%$ of the included patients (9). Some articles mention sentinel nodes missed on both planar lymphoscintigraphy and SPECT/CT in from $3 \%$ to $31 \%$ of the patients $(3,9,10)$. Two reports describe percentages of metastatic sentinel nodes detected on SPECT/CT only. In melanoma, this percentage was 3\% of all studied patients, whereas in a report focusing on bladder cancer this percentage was $83 \%(3,11)$. Other investigators describe an incidence of $0 \%-5 \%$ for patients with initially missed metastatic nodes detected only on SPECT/CT $(6,8,9)$. One study focused on overweight breast cancer patients and reported that SPECT/CT detected significantly more sentinel nodes than did planar imaging (91\% vs. $78 \%$ ) in this patient group (19).

Several studies demonstrated improved anatomic localization of sentinel nodes by SPECT/CT in $98 \%-100 \%$ of the examined patients $(3,11,12)$. A study performed on 157 breast cancer patients specified that additional sites of lymphatic drainage detected only on SPECT/CT were found in the axilla $(n=23)$, in the internal mammary chain $(n=5)$, within the breast $(n=2)$, and in the interpectoral fossa $(n=$ 3) (9). With use of a hybrid SPECT/CT device, an intraoperative detection rate of as high as $100 \%$ has been reported for sentinel nodes $(5,11)$. One study showed the detection rate to be $85 \%$ with only the blue dye method but $100 \%$ when both planar and SPECT/CT images were obtained (5).

Most investigators who used SPECT/CT as an additional imaging tool in lymphatic mapping did not formulate their indications. We limited the use of SPECT/CT to difficult and unusual cases because we believe planar lymphoscintigraphy is an excellent preoperative mapping technique for most patients. On the basis of our experience in the first 20 patients, we added nonvisualization as a new indication, because SPECT/CT visualized drainage in patients whose planar images did not reveal a sentinel node. In addition, we believe that the added costs and extra time for SPECT/ $\mathrm{CT}$ are more justified when the procedure is used for this new indication.

\section{CONCLUSION}

SPECT/CT was useful for finding the exact anatomic location of sentinel nodes and in detecting additional sites 
of drainage. These advantages facilitate surgical exploration and eventually lead to more accurate staging. In the future, SPECT/CT may also obviate preoperative skin marking and may replace delayed lateral planar imaging. Whether SPECT/CT should be used on all patients or only for specific indications needs to be studied further.

\section{ACKNOWLEDGMENTS}

We thank Christel Feenstra and colleagues of the Department of Nuclear Medicine for their technical support in the acquisition and reconstruction of SPECT/CT images. Marie-Jeanne Vrankcen-Peeters and Hester Oldenburg operated on most of the breast cancer patients mentioned in this pilot study and helped with collecting the required data.

\section{REFERENCES}

1. Even-Sapir E. Sentinel node scintigraphic mapping using SPECT/CT. In: Israel O, Goldsmith S, eds. Hybrid SPECT/CT Imaging in Clinical Practice. New York, NY: Taylor and Francis Group; 2006:121-139.

2. Keidar Z, Israel O, Krausz Y. SPECT/CT in tumor imaging: technical aspects and clinical applications. Semin Nucl Med. 2003;33:205-218.

3. Kretschmer L, Altenvoerde G, Meller J, et al. Dynamic lymphoscintigraphy and image fusion of SPECT and pelvic CT-scans allow mapping of aberrant pelvic sentinel lymph nodes in malignant melanoma. Eur J Cancer. 2003;39:175-183.

4. Kim W, Menda Y, Willis J, Bartel T, Graham M. Use of lymphoscintigraphy with SPECT/CT for sentinel node localization in a case of vaginal melanoma. Clin Nucl Med. 2006;31:201-202.

5. Ishihara T, Kaguchi A, Matsushita S, et al. Management of sentinel lymph nodes in malignant skin tumors using dynamic lymphoscintigraphy and the singlephoton-emission computed tomography/computed tomography combined system. Int J Clin Oncol. 2006;11:214-220.
6. Wagner A, Schicho K, Glaser C, et al. SPECT-CT for topographic mapping of sentinel lymph nodes prior to gamma probe-guided biopsy in head and neck squamous cell carcinoma. J Craniomaxillofac Surg. 2004;32:343-349.

7. Even-Sapir E, Lerman H, Lievshitz G, et al. Lymphoscintigraphy for sentinel node mapping using a hybrid SPECT/CT system. J Nucl Med. 2003;44:14131420.

8. Khafif A, Schneebaum S, Fliss DM, et al. Lymphoscintigraphy for sentinel node mapping using a hybrid single photon emission CT (SPECT)/CT system in oral cavity squamous cell carcinoma. Head Neck. 2006;28:874-879.

9. Lerman H, Metser U, Lievshitz G, Sperber F, Shneebaum S, Even-Sapir E. Lymphoscintigraphic sentinel node identification in patients with breast cancer: the role of SPECT-CT. Eur J Nucl Med Mol Imaging. 2006;33:329-337.

10. Kizu H, Takayama T, Fukuda M, et al. Fusion of SPECT and multidetector CT images for accurate localization of pelvic sentinel lymph nodes in prostate cancer patients. J Nucl Med Technol. 2005;33:78-82.

11. Sherif A, Garske U, de la Torre M, Thorn M. Hybrid SPECT-CT: an additional technique for sentinel node detection of patients with invasive bladder cancer. Eur Urol. 2006;50:83-91.

12. Schillaci O, Danieli R, Manni C, Simonetti G. Is SPECT/CT with a hybrid camera useful to improve scintigraphic imaging interpretation? $\mathrm{Nucl}$ Med Commun. 2004;25:705-710.

13. Morton DL, Wen D-R, Wong JH, et al. Technical details of intraoperative lymphatic mapping for early stage melanoma. Arch Surg. 1992;127:392-399.

14. Nieweg OE, Jansen L, Valdés Olmos RA, et al. Lymphatic mapping and sentinel lymph node biopsy in breast cancer. Eur J Nucl Med. 1999;26(suppl):S11-S16.

15. Hasegawa BH, Stebler B, Rutt BK, et al. A prototype high-purity germanium detector system with fast photon-counting circuitry for medical imaging. Med Phys. 1991;18:900-909.

16. Lang TF, Hasegawa BH, Liew SC, et al. Description of a prototype emissiontransmission computed tomography imaging system. J Nucl Med. 1992;33: 1881-1887.

17. Estourgie SH, Tanis PJ, Nieweg OE, Valdés Olmos RA, Rutgers EJTh, Kroon BBR. Should the hunt for internal mammary chain sentinel nodes begin? An evaluation of 150 breast cancer patients. Ann Surg Oncol. 2003;10:935-941.

18. Van Rijk MC, Tanis PJ, Nieweg OE, et al. Clinical implications of sentinel nodes outside the axilla and internal mammary chain in patients with breast cancer. J Surg Oncol. 2006;94:281-286.

19. Lerman H, Lievshitz G, Zak O, Metser U, Schneebaum S, Even-Sapir E. Improved sentinel node identification by SPECT/CT in overweight patients with breast cancer. J Nucl Med. 2007;48:201-206. 